\title{
Risk factors for lower respiratory tract infection associated with tracheobronchial foreign body aspiration in children
}

\author{
Fuzhi $\operatorname{Lin}^{1}$, Rongrong $\mathrm{Wu}^{1}$, Bin $\mathrm{Xu}^{1}$, Jia Liu ${ }^{1}$, Jing $\mathrm{Bi}^{1}$, and Yong $\mathrm{Fu}^{1}$ \\ ${ }^{1}$ Zhejiang University School of Medicine Children's Hospital
}

February 19, 2021

\begin{abstract}
Background: Tracheobronchial foreign body aspiration (TFBA) is a major cause of death in children. There are few reports about lower respiratory tract infection (LRTI) caused by TFBA. This study collected the TFBA in our hospital to analyze the LRTI and to determine its risk factors. Methods: A total of 194 children who were performed TFBA extraction in The Children's Hospital of Zhejiang University School of Medicine between June 2019 and April 2020 were enrolled. The clinical data, cervicothoracic CT and operation records were collected. Chi-square test, rank sum test and multivariate logistics regression analysis were applied. Results: The incidence of LRTI was $46.6 \%, 68.6 \%$ and $68.6 \%$ respectively when retention time was within 24 hours, 1 week and more than 1 week. Cervicothoracic CT showed embedding site in and above trachea in 24 cases, in main bronchus in 134 cases, in segmental bronchus and below in 36 cases. Cervicothoracic CT showed no obvious abnormality in 60 cases, obstructive emphysema or atelectasis in 77 cases, obstructive pneumonia in 50 cases, and the rest in 7 cases. Cervicothoracic CT diagnosis and retention time of TFBA were different between LRTI group and non-LRTI group in univariate analysis. There was a correlation between the retention time of TFBA and LRTI in multivariate logistics regression analysis. Conclusionss: The incidence of LRTI caused by TFBA is high. There was no difference in the incidence of LRTI between organic and inorganic TFBA. The retention time of TFBA is an independent risk factor for LRTI.
\end{abstract}

Risk factors for lower respiratory tract infection associated with tracheobronchial foreign body aspiration in children

Fuzhi Lin MM, Rongrong Wu MB, Bin Xu MM, Jia Liu MM, Jin Bi MM, Yong Fu MD

Department of Otorhinolaryngology Head and Neck Surgery, Children's Hospital, Zhejiang University School of Medicine, Hangzhou 310051, Zhejiang Province, China

Correspondence

Yong Fu, MD, Department of Otorhinolaryngology Head and Neck Surgery, Children's Hospital, Zhejiang University School of Medicine, Hangzhou 310051, Zhejiang Province, China. E-mail: fyent@163.com

\section{Abstract}

Background:Tracheobronchial foreign body aspiration (TFBA) is a major cause of death in children. There are few reports about lower respiratory tract infection (LRTI) caused by TFBA. This study collected the TFBA in our hospital to analyze the LRTI and to determine its risk factors.

Methods: A total of 194 children who were performed TFBA extraction in The Children's Hospital of Zhejiang University School of Medicine between June 2019 and April 2020 were enrolled. The clinical data, cervicothoracic CT and operation records were collected. Chi-square test, rank sum test and multivariate logistics regression analysis were applied. 
Results: The incidence of LRTI was $46.6 \%, 68.6 \%$ and $68.6 \%$ respectively when retention time was within 24 hours, 1 week and more than 1 week. Cervicothoracic CT showed embedding site in and above trachea in 24 cases, in main bronchus in 134 cases, in segmental bronchus and below in 36 cases. Cervicothoracic CT showed no obvious abnormality in 60 cases, obstructive emphysema or atelectasis in 77 cases, obstructive pneumonia in 50 cases, and the rest in 7 cases. Cervicothoracic CT diagnosis and retention time of TFBA were different between LRTI group and non-LRTI group in univariate analysis. There was a correlation between the retention time of TFBA and LRTI in multivariate logistics regression analysis.

Conclusionss: The incidence of LRTI caused by TFBA is high. There was no difference in the incidence of LRTI between organic and inorganic TFBA. The retention time of TFBA is an independent risk factor for LRTI.

\section{Key words}

Tracheobronchial; Foreign body; Infection; CT; Type; Children.

Abbreviations: TFBA, Tracheobronchial foreign body aspiration; LRTI, lower respiratory tract infection

\section{INTRODUCTION}

Tracheobronchial foreign body aspiration (TFBA) often occurs in children, and especially in children under 3 years old ${ }^{1}$. They tend to have hypoplasia of molars, poor masticatory function, weak choking ability, and they are easy to cry during dining ${ }^{2}$. In a result, the incidence of them is higher. TFBA is also a major cause of death among children ${ }^{3}$. Although the survival rate of TFBA has been greatly improved since the development of rigid bronchoscope in 19 th century ${ }^{4}$, it is still a major challenge for otorhinolaryngology. The trachea of children is smaller than adults, which leads to a more limited operative field under rigid bronchoscope. Also, TFBA can easily lead to varied degrees of damage in tracheal mucosa ${ }^{5}$,such as mucosal follicular hyperplasia, hyperemia and swelling, inflammatory stenosis of bronchial lumen, blockage of bronchial lumen by purulent secretions, all resulting in an even worse operative field. Therefore, we hope to find a clinically available indicator to predict the high incidence of lower respiratory tract infection(LRTI), so that the operator can be well prepared before the operation.

Emphysema, atelectasis and pneumonia are common complications of $\mathrm{TFBA}^{6}$, however, there are few studies on LRTI caused by TFBA. Studies are mainly focused on bacterial culture ${ }^{7,8}$. According to previous studies, there was no correlation between the type of TFBA and LRTI in animal studies, whereas the retention time of TFBA was related ${ }^{9}$. A research reported that type, shape and the retention time of TFBA and age were associated with LRTI $^{10}$. Therefore, we retrospectively analyzed the children who were applied TFBA extraction in our hospital in order to determine the occurrence of LRTI and analyze its risk factors.

\section{Materials and methods}

\subsection{Collection of clinical data}

This study was approved by the ethics committee of The Children's Hospital of Zhejiang University School of Medicine, and all patients were informed of the relevant research and provided their written consent. A total of 194 children who were operated TFBA surgery in The Children's Hospital of Zhejiang University School of Medicine from June 01, 2019 to April 30, 2020 was researched. The general characteristic, clinical symptoms, operative records, cervicothoracic CT, type and retention time of TFBA and weight were collected. The diagnosis of cervicothoracic CT was made by two senior attending doctors in our department according to clinical history. LRTI is defined as infection and inflammation of trachea, main bronchus and lung bronchi. Imaging basis includes preoperative cervicothoracic CT and intraoperative rigid bronchoscopy ${ }^{10}$. Clinical diagnosis is based on relevant clinical guidelines ${ }^{11,12}$.

\subsection{Inclusion criteria and exclusion criteria}

Inclusion criteria: 1) performed surgery in our hospital and confirmed the presence of TFBA; 2) preoperative 
examination can be collected; 3) the age was under 14 years old. Exclusion criteria: 1) incomplete clinical data; 2) no TFBA was found during operation; 3) refused to do the operation; 4) with acute or chronic infection caused by other confirmed reasons; 5) treated with antibiotics within 72 hours before operation.

\subsection{Statistical methods}

The Kolmogorov-Smirnov method was used to test the normal distribution. The rank sum test was used to analyze the difference in continuous variables that are in non-normal distribution. The chi-square test was used to analyze the difference in classified variables. The variables with statistical differences were included in multivariate logistics regression analysis. When $\mathrm{P}<0.05$, the differences were considered to be statistically significant. The statistical software was SPSS24.0.

\section{Results}

A total of 194 cases of TFBA were included in this study, 120 males and 74 females with a median age of 1.54 years $(0.6-12.58$ years). The types of TFBA were inorganic $(\mathrm{n}=7)$, other organic $(\mathrm{n}=33)$, nuts $(\mathrm{n}$ $=143)$ and legumes $(\mathrm{n}=11)$. Cervicothoracic CT showed TFBA embedded in trachea and above trachea in 24 cases, in left and right main bronchus in 134 cases (left bronchus 87 cases, right bronchus 47 cases), in segmental bronchus and below in 36 cases (left segmental bronchus 6 cases, right segmental bronchus 30 cases). Cervicothoracic CT showed no obvious abnormality in 60 cases, obstructive emphysema or atelectasis in 77 cases, obstructive pneumonia 50 cases, and the rest 7 cases (pleural effusion, mediastinal displacement, subcutaneous emphysema, pneumothorax and mediastinal emphysema). The general characteristics are showed in Table 1.

We conducted a univariate analysis on risk factors mentioned in previous literature and clinically considered possible. The results showed that there was a significant difference in cervicothoracic CT diagnosis and the retention time of TFBA between LRTI group and non-LRTI group. The results of univariate analysis are showed in Table 2 .

Cervicothoracic CT diagnosis and the retention time of TFBA were included in multivariate logistics regression analysis. We found that there was a correlation between the retention time of TFBA and LRTI, while no correlation was found between cervicothoracic CT diagnosis and LRTI. The results of multivariate logistics analysis are showed in Table 3 .

\section{Discussion}

TFBA is a clinical emergency and a major cause of death in children ${ }^{13}$. At present, the most effective way to remove TFBA is through the rigid bronchoscope as soon as possible. The most important diagnosis is based on the history of foreign body choking ${ }^{14}$.

LRTI is easily caused by TFBA. In this study, most children had purulent secretions. Previous studies reported that TFBA can cause obstructive emphysema and pneumonia ${ }^{15}$ based on preoperative CT. However, as a LRTI, acute bronchitis with no positive signs in CT is often ignored. Limited studies reported LRTI caused by TFBA, while they are mainly focused on bacterial culture ${ }^{7,8}$. Only one study reported the risk factors for $\mathrm{LRTI}^{10}$. As a result, the situation of LRTI cannot be fully showed.

In clinical practice, LRTI plays an important role in intraoperative operation and disease prognosis. A study reported that TFBA with LRTI have a longer hospital stay ${ }^{10}$. Therefore, we hope to find a relevant indicator to predict the occurrence of LRTI for operators so that they can pay more attention to prepare the operation. This study collected the children who were applied TFBA extraction and analyzed the risk factors for LRTI.

We used to have a clinical understanding that organisms are more likely to cause inflammation and suppuration in tracheobronchial tissues than non-organisms, especially plants ${ }^{16}$. In addition, it is reported that the type of TFBA was an independent risk factor for LRTI ${ }^{10}$. However, recent animal experiments found 
that there was no difference in tracheal damage between organic and inorganic foreign bodies ${ }^{9}$. Similarly, we found that there was no statistical difference in the incidence of LRTI among legumes, nuts, other organisms and non-organisms. We also found a correlation between the retention time of TFBA and LRTI. In our study the incidence of LRTI was $46.6 \%, 68.6 \%$ and $68.6 \%$ respectively with the retention time of within 24 hours, one week and more than one week, which was consistent with the results of a previous animal experiment ${ }^{9}$. In this animal experiment, it was found that most of the TFBA could cause tracheal damage within 5 days, and pneumonia more than 5 days. No matter in animal experiments or clinical findings, LRTI caused by TFBA could be aggravated with the increase of time.

We speculate that there are three main reasons for the differences between previous clinical understandings and our study. Firstly, the family members have a significantly higher understanding of TFBA than before, and the visiting time is significantly shortened. Secondly, CT has been more popular which increases the accuracy and timeliness of diagnosis. Thirdly, it may need time to realize the impact of the type of TFBA on trachea tissue. This suggests that no matter what type of TFBA is or where the TFBA is embedded, the longer the time is, the more serious the LRTI is. Therefore, we suggest that for children with long retention time of TFBA, the operators should be vigilant and fully prepared before operation. They should also inform the parents that the operation can be more difficult and complicated than those with short retention time.

In a report of LRTI caused by TFBA ${ }^{10}$, type, shape, the retention time of TFBA and age were independent risk factors for LRTI. Analyzed all the related risk factors in our study, except for the shape of TFBA, there was a correlation between the retention time of TFBA and LRTI, while there is no correlation between age, the type of TFBA and LRTI. We speculate that the main reason is that the family members have different understanding of TFBA, which leads to the difference in visiting time. In the above study, the median retention time of TFBA is 15 days, while it's only 1 day in our study. It is controversial whether there is a difference in the degree of trachea inflammation caused by the type of TFBA. Although it has been reported that the intratracheal granulation in TFBA is related to the degree of TFBA oil release ${ }^{17}$, no correlation was found in animal experiments ${ }^{9}$. As for age, we speculate that the immunity of younger children is relatively weak so that they are easier to get LRTI when encounter the same TFBA. However, it needs time to show this difference.

No indicator of LRTI caused by TFBA was found in clinical symptoms. When choked by foreign body, the more severe the cough and asthma is, which indicates that the TFBA is too large, the larynx and trachea spasm caused by it is obvious and the blockage of foreign body is serious, the more dangerous the process of choking is. However, tracheal mucosal inflammation needs time to happen, these clinical symptoms cannot predict the incidence of LRTI. Despite of this fact, they reflect the severity of the disease. Operations should be done as soon as possible for children with obvious symptoms.

There is no specific standard for the subgroup classification of TFBA complications. According to previous literature, we found that there is similar classification for obstructive pneumonia and atelectasis in non-small cell lung cancer, which can be used as a reference. Firstly, non-small cell lung cancer respectively showed segmental and lobar local emphysema or atelectasis in incomplete or complete blockage of the bronchial lumen. Secondly, in the TNM stage of non-small cell lung cancer ${ }^{18}$,non-small cell lung cancer with obstructive pneumonia or partial or whole atelectasis is classified as T2 stage. In this guideline, atelectasis and emphysema are regarded as the same element. Furthermore, in previous prognostic studies of non-small cell lung cancer, preoperative obstructive pneumonia and atelectasis were also put into one group without discussed separately ${ }^{19}$ when the overall survival rate and recurrence-free survival rate of lung cancer were compared. To sum up, we drew lessons from the classification method of non-small cell lung cancer, and put atelectasis and emphysema into the same group, considering them as different manifestations of the same type. Based on this method, we further divided atelectasis or emphysema and pneumonia into different subgroups. Combined with the clinical criticality, we classified the mediastinal emphysema, pneumothorax, subcutaneous emphysema, severe pulmonary inflammation, such as pleural effusion, and mediastinal displacement as a group. In univariate analysis, we found that the worse the clinical condition was indicated by cervicothoracic CT, the higher the incidence of LRTI would be. However, in multivariate analysis, there 
was an overlapping relationship between cervicothoracic CT diagnosis and the retention time of TFBA, suggesting that the longer the retention time was, the more serious the LRTI would be. The cervicothoracic CT diagnosis was not related to LRTI.

This study is limited in the following ways. Firstly, the number of cases included is small, and it is a single center study, which needs to be supplemented by a larger sample size. Secondly, this study only includes a single race. It is not clear whether there is a correlation between race and LRTI. Thirdly, the educational background of the families and their attention paid to children when they are eating may be risk factors for LRTI. Due to the lack of these information, this study is limited in its retrospective way.

\section{Conclusions}

The incidence of LRTI caused by TFBA is very high. However, the type and history of TFBA, cervicothoracic CT diagnosis, embedded location, clinical symptoms, weight, sex and age cannot predict its occurrence. There is no significant difference in the incidence of LRTI between organic and inorganic TFBA. Symptoms, cervicothoracic CT diagnosis and embedded location can only indicate the severity of the TFBA. The retention time of TFBA is the only indicator of the LRTI. Children with TFBA for a long retention time should be paid more attention in clinic.

\section{ACKNOWLEDGMENTS}

We thank the patients and doctors who participated in this study.

\section{CONFLICTS OF INTEREST}

The authors declare no conflict of interest.

\section{References}

1. Baharloo F, Veyckemans F, Francis C, Biettlot MP, Rodenstein DO. Tracheobronchial foreign bodies: presentation and management in children and adults. CHEST. 1999;115:1357-1362.

2. Violence COI, Prevention P. Prevention of choking among children. PEDIATRICS. 2010;125:601-607.

3. Johnson K, Linnaus M, Notrica D. Airway foreign bodies in pediatric patients: anatomic location of foreign body affects complications and outcomes. PEDIATR SURG INT. 2017;33:59-64.

4. CLERF, Louis H. Historical aspects of foreign bodies in the air and food passages. SOUTH MED J. 1975;68:1449-1454.

5. Xin LH, Wang Z, Yu HC. Clinical value of electronic bronchoscope diagnosis and treatment of children tracheal bronchus foreign body. China Journal of Endoscopy. 2016;22:43-46.

6. Mu LC, He P, Sun DQ. The causes and complications of late diagnosis of foreign body aspiration in children. Arch Otolaryngol. 1991;117:876-879.

7. Gruber M, Meer GVD, Ling B, Barber C, Mahadevan M. The bacterial species associated with aspirated foreign bodies in children. AURIS NASUS LARYNX. 2018;45:598-602.

8. Liu XM, Zhou L, Wang ZH, Jiang Y, Qu ZH. Multiple polymerase chain reaction detection results of pathogenic bacteria in children with bronchial foreign body and lower respiratory tract infection. Journal of Precision Medicine. 2020;35:250-252.

9. Hughes CK, Christensen CL, Maturo SC, O'Connor PR, Dion GR. Organic vs. Inorganic Tracheobronchial Airway Foreign Body Aspiration: Does Type/Duration Matter? The Laryngoscope. 2020.

10. Zhong B, Sun SL, Du JT, Deng D, Liu F, Liu YF, Liu SX, Chen F. Risk factors for lower respiratory tract infection in children with tracheobronchial foreign body aspiration. Medicine (Baltimore). 2019;98: e14655. 
11. Chinese Medical Association, Chinese Medical Journals Publishing House, Chinese Society of General Practice, the Respiratory Infection Assembly of Chinese Thor, Editorial Board of Chinese Journal of General Prac, Expert Group of Guidelines for Primary Care of Res. Guideline for primary care of acute tracheobronchitis(2018). Chinese Journal of General Practitioners. 2019;18:314-317.

12. National Health Commission of the People's Republic of China, State Administration of Traditional Chinese Medicine. Guideline for diagnosis and treatment of community-acquired pneumonia in Children (2019 version). Chin J Clin Infect Dis. 2019;12:6-13.

13. Karako F, Karada B, Akbenliolu C, Ersu R, Yldzeli B, Yüksel M, Dal E. Foreign body aspiration: what is the outcome? PEDIATR PULM. 2010;34:30-36.

14. Salih AM,Alfaki M,Alam-Elhuda DM,FO Medicine,International University of Africa,MV Medicine,UO Edinburgh,Ribat University and Central Police Hospitals,NR University,UO Khartoum. Airway foreign bodies: A critical review for a common pediatric emergency. World J. Emerg. Med. 2016;7:5-12.

15. Adegboye VO, Osinowo O, Adebo OA. Bronchiectasis consequent upon prolonged foreign body retention. Cent. Afr. J. Med. 2003;49:53.

16. Xue G, Shang XL, Lin YT, Xu GG, Wu LX, Zhang QJ. Tracheobronchial foreign bodies in children:clinical analysis of 3018 cases. Chinese Journal of Otorhinolaryngology-Skull Base Surgery. 2008;14:372374.

17. Huang Z, Zhou A, Zhang J, Xie L, Li Q. Risk factors for granuloma formation in children induced by tracheobronchial foreign bodies. INT J PEDIATR OTORHI. 2015;79:2394-2397.

18. Rusch VW, Chansky K, Kindler HL, Nowak AK, Pass HI, Rice DC, Shemanski L, Galateau-Salle F, McCaughan BC, Nakano T, et al. The IASLC lung cancer staging project: Proposals for revision of the TNM stage groupings in the forthcoming (eighth) edition of the TNM Classification for lung cancer. J THORAC ONCOL. 2016;11:39-51.

19. Pang Z, Ding N, Dong W, Ni Y,Zhang T,Qu X,Du J,Liu Q. Prognostic effects of preoperative obstructive pneumonitis or atelectasis and comparison with tumor size in non-small cell lung cancer. J THORAC DIS. 2017;9:768.

\section{Hosted file}

E-table 1.docx available at https://authorea.com/users/396775/articles/509856-risk-factorsfor-lower-respiratory-tract-infection-associated-with-tracheobronchial-foreign-bodyaspiration-in-children

\section{Hosted file}

E-table 2.docx available at https://authorea.com/users/396775/articles/509856-risk-factorsfor-lower-respiratory-tract-infection-associated-with-tracheobronchial-foreign-bodyaspiration-in-children

\section{Hosted file}

E-table 3.docx available at https://authorea.com/users/396775/articles/509856-risk-factorsfor-lower-respiratory-tract-infection-associated-with-tracheobronchial-foreign-bodyaspiration-in-children 